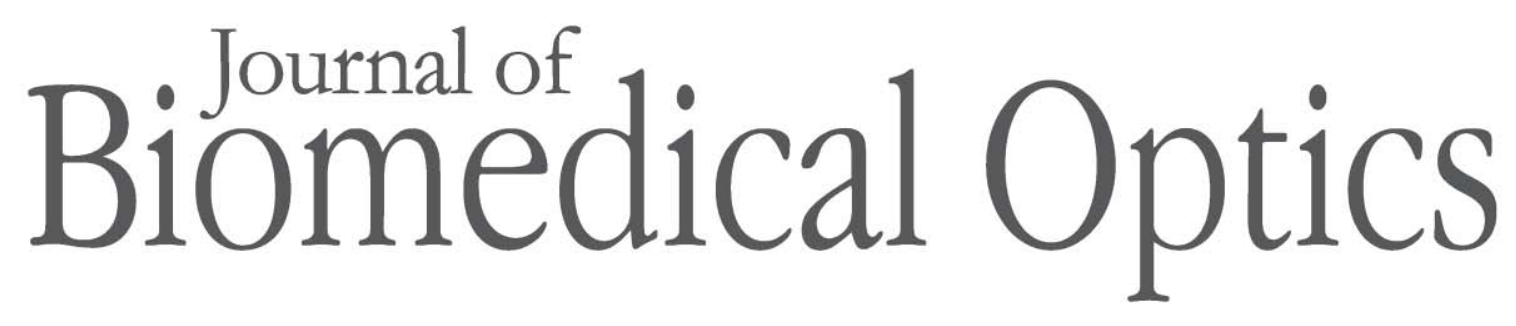

SPIEDigitalLibrary.org/jbo

\title{
Mechanical design of a power-adjustable spectacle lens frame
}

Asuncion Zapata

Sergio Barbero 


\title{
Mechanical design of a power-adjustable spectacle lens frame
}

\author{
Asuncion Zapata and Sergio Barbero \\ Instituto de Optica, CSIC, Madrid, 28006 Spain
}

\begin{abstract}
Power-adjustable spectacle lenses, based on the Alvarez-Lohmann principle, can be used to provide affordable spectacles for subjective refractive errors measurement and its correction. A new mechanical frame has been designed to maximize the advantages of this technology. The design includes a mechanism to match the interpupillary distance with that of the optical centers of the lenses. The frame can be manufactured using low cost plastic injection molding techniques. A prototype has been built to test the functioning of this mechanical design. () 2011 Society of Photo-Optical Instrumentation Engineers (SPIE). [DOI: 10.1117/1.3582160]
\end{abstract}

Keywords: Alvarez-Lohmann lens; self-adjustable lens; spectacle lens design.

Paper 10543RR received Oct. 6, 2011; revised manuscript received Apr. 4, 2011; accepted for publication Apr. 4, 2011; published online May 19, 2011.

\section{Introduction}

Published surveys estimate ${ }^{1}$ that there are around 153 million visually impaired people ( 8 million considered blind) because of uncorrected refractive errors. These errors are commonly corrected with monofocal spectacle lenses. However political, socio-economical and technological drawbacks prevent these people from having access to affordable refraction and spectacle provision. Several authors ${ }^{2-5}$ have proposed new technological solutions using the concept of power-adjustable lenses to combat this major medical and social problem.

The power-adjustable spectacle is able to measure and correct different refractive errors using the same spectacle design, therefore reducing costs, both in terms of human and economic resources. Two techniques have been proposed: one using liquidfilled variable focus lenses ${ }^{2}$ and the other applying the AlvarezLohmann principle. ${ }^{3-5}$

The Alvarez-Lohmann principle ${ }^{3,6}$ states that when two lenses with cubic-type surfaces are slid laterally, a power change, proportional to the amount of shift, is induced (see, e.g., Barbero ${ }^{7}$ and references therein). These lateral shifts only change the sphere component of refraction. However, it could be possible to modify the cylinder component with rotation of the lenses around the optical axis, ${ }^{8}$ although such combination of rotational and lateral movements would imply rather complex spectacle lens frames. These have not been considered here.

A major challenge in using Alvarez-Lohmann lenses is designing a mechanical mechanism and attaching it to the frame, in a manner that permits lateral movements.

Previous works, in patent literature, have proposed different mechanical designs. Some arrange the lateral movement along the eyelid-cheek (vertical) direction, but others along the nasal-temporal (horizontal) direction. ${ }^{9,10}$ They all have in common that only one lens is moved, keeping the other in a fixed position. One single exception is the model proposed in Patent No. WO-2006/098618, ${ }^{9}$ where both lenses are moved

Address all correspondence to: Sergio Barbero, School of Optometry, 800 East Atwater Avenue, Bloomington, Indiana 47405-3680. Tel: 812-3341658; Fax: 812-8557445; E-mail: sergio.barbero@io.cfmac.csic.es. in opposite directions, although the mechanism does not ensure that the lenses move exactly the same amount.

An example of the use of vertical movement is demonstrated in Patent No. WO-2008/002131, ${ }^{11}$ where the movement is obtained by means of a vertical worm screw integrated in the frame, with a mechanism locking the moving lens at its final position. In Patent No. US-7325922, ${ }^{10}$ an actuating lever slides the lenses along a guiding groove. In Patent No. WO-2006/098618 a thread helix-like element transforms a vertical into a horizontal movement through a cam element attached to a mechanized groove in each lens.

As an alternative to the linear lateral movements, the lenses could be adjusted using a rotational mechanism. One of the lens would rotate on the other, relative to an axis perpendicular to the viewing direction. Examples are: Patent No. US$2007 / 0030440,{ }^{12}$ where the rotational device is located within the frame bridge, whereas in Patent No. US-7325922, ${ }^{10}$ it is located within the frame sides. It is important to note that the axis of rotation in these devices is not matched with the optical axis of both lenses. This is why these models, though using rotational movements, cannot be used for the correction of astigmatism.

Finally, Patent No. WO-2009/140255 ${ }^{13}$ proposes an original solution, making use of a magnetic attachment.

The main drawback of these designs is that they only move one lens of the pair (except in Patent No. WO-2006/098618 ${ }^{9}$ ). When only one lens is moved, it generates a lateral shift of the optical center, which introduces prismatic errors. ${ }^{7}$ Moreover, in spectacle lenses, the interpupillary distance must be equal to that found between the optical centers of the lenses to avoid horizontal prismatic errors. To meet this requirement, lenses are usually cut in order to place the optical center in the pupil center location, for a given viewing direction. This procedure could also be applied to Alvarez-Lohmman lenses but this would reduce some of the low cost benefits of this type of technology. A solution to this problem is to design an additional mechanical device, which allows the two pair of lenses to be moved horizontally.

1083-3668/2011/16(5)/055001/6/\$25.00 (C) 2011 SPIE 
The goal of this work was to design a new type of frame for Alvarez-Lohmann power-adjustable spectacle lenses, overcoming the drawbacks of the aforementioned mechanical designs. It also considers many other aspects not discussed in previous works, such as: dimension constraints, mechanical stability, and manufacturing issues.

The minimum required lateral movement between lenses is given by the smallest refraction prescription. This implicates a micromechanical design. To determine, with precision, the amount of lateral shift applied to the lenses, an external imprinted rule with a millimetric scale can be used.

Our design considers face anthropometry constraints, as described in several papers ${ }^{14,15}$ and also takes into account mechanical stability criteria in order to offer a long lasting product. The frame is composed of several pieces designed to be assembled manually. The modular feature of the frame offers the advantage of easy replacement of any broken pieces and easy cleaning of the lenses and frame. Finally, the spectacle lens frame has been designed to be manufactured using plastic injection molding techniques, thus ensuring a low cost technology.

\section{Mechanical Design Description}

Spectacle lens mounts are typically composed of the following elements: ${ }^{16}$ the rims (contours of lens holders), the bridge joining the rims, the frame sides which fix the spectacle frame to the ears, and the nose pad bearing the weight of the spectacles on the nose. These different elements must ensure that the spectacles fit facial anthropometry. ${ }^{14,15}$

The spectacles frame, presented here, integrates the nose pads and the bridge into a single complex structure, which includes two mechanisms to achieve the lens movements. This fixed structure (plotted in Fig. 1) is a solid piece whose width is set by the frontal horizontal dimension of the spectacle frame.

There are two mobile structures. One moves the two pairs of lenses horizontally, which is necessary to match the interpupillary distance with that of the optical centers. The other moves the lenses vertically to change the optical power.

It is recommended that if the weight of the spectacles is more than $25 \mathrm{~g}$, the minimum contact area of the nose pads must be $200 \mathrm{~mm}^{2}$ (BS-EN-ISO-12870). ${ }^{17}$ According to this recommendation, and considering that our spectacle weight exceeds $25 \mathrm{~g}$, we designed nose pads with a contact area of $205 \mathrm{~mm}^{2}$ [Fig. 1(a)]. In addition, the nose pads were designed based on typical values of the spay and frontal angles reported in the literature. ${ }^{14,15}$

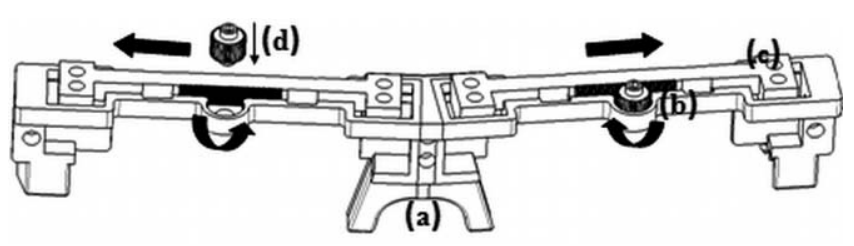

Fig. 1 Fixed structure comprising: (a) nose pads, (b) pinions, and (c) racks. (d) Pinions assembly direction into the fix structure.

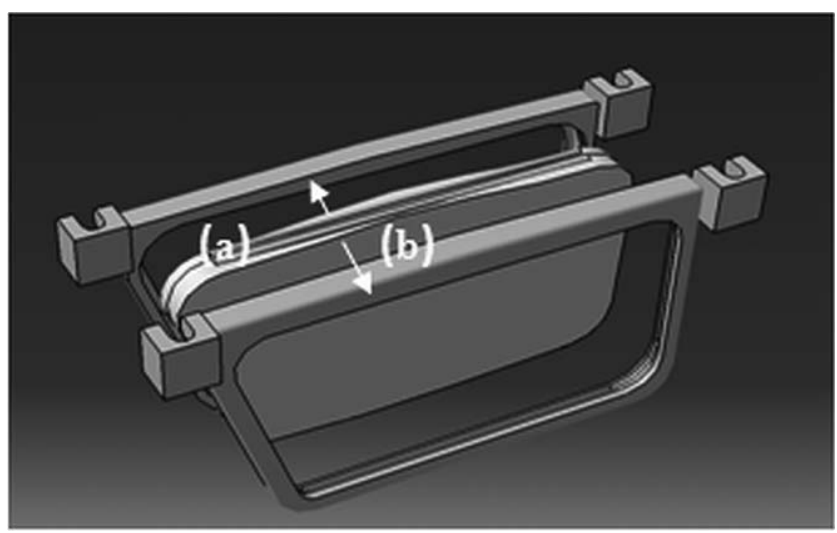

Fig. 2 Lens holders and lenses. (a) Lens edge. (b) Mounting direction of lenses into the lens holders.

\subsection{Horizontal Movement Mechanism}

The system comprises two pinion-rack assemblies that move the two pair of lens holders. When the pinion [Fig. 1(b)] turns, the pinion teeth exert pressure on those of the rack [Fig. 1(c)], causing a linear horizontal displacement of the lens holders in two possible directions indicated in Fig. 1. The pinions, turned with the help of a screwdriver, have 36 teeth with a thread pitch of $0.5 \mathrm{~mm}$ and a module of 0.159 . The rack has the same module. This mechanism provides horizontal movements of $0.5 \mathrm{~mm}$ per $9.5^{\circ}$ pinion rotations. Each side's maximum amount of horizontal movement is $2 \mathrm{~mm}$. Therefore, this mechanism can eliminate differences of up to $4 \mathrm{~mm}$ between the interpupillary distance and that found between optical centers. This range should be sufficient to correct the discrepancies found in a reasonable number of subjects of specific race and gender. ${ }^{18}$

\subsection{Vertical Movement Mechanism}

The front and rear lenses are mounted in separate lens holders [Fig. 2]. Their movement is achieved using a set of specially designed screws, which are threaded into the racks of the horizontal movement component [Fig. 3]. Two screws move each lens holder to achieve a stable vertical movement [Fig. 4] for each pair of lenses.

These screws [Fig. 2(a)] are composed of two different parts. The upper part [Fig. 5(a)] is threaded (M3×13) while the lower part is rounded with a circular groove [Fig. 5(b)]. The lens holder is held in this groove. As the screw moves upward the circular groove creates a displacement of the piece where the lens holder and the screw are joined (from now on designated as screw holder) [Fig. 5]. The screws have a thread pitch of

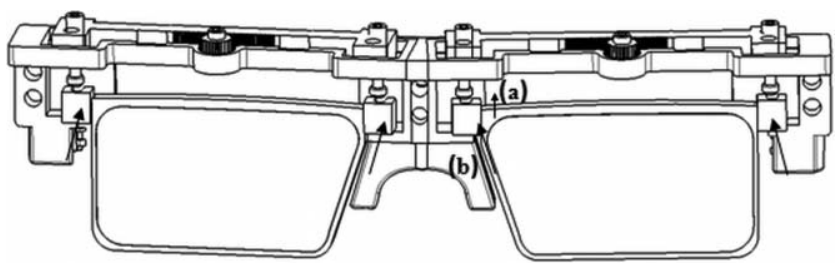

Fig. 3 Lens holders and fixed structure. (a) Screws holding the lens holders. (b) Mounting direction of lens holders. 


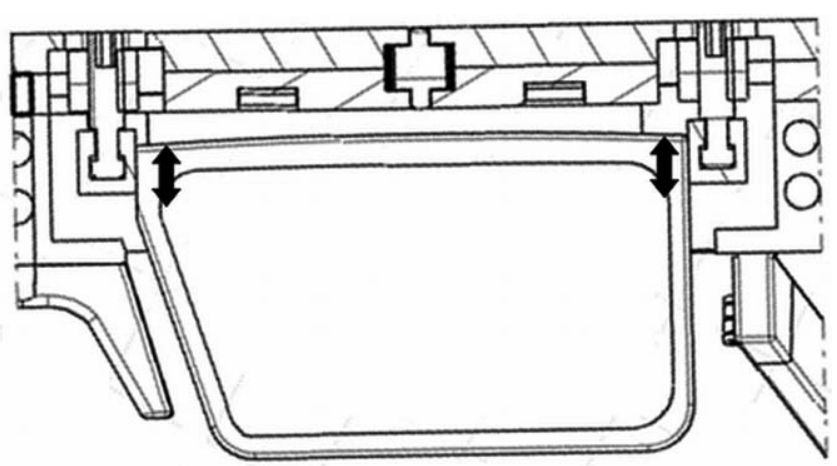

Fig. 4 Two screws moving vertically each lens.

$0.35 \mathrm{~mm}$. Therefore, when the screw turns $360^{\circ}$, the lenses are moved vertically $0.35 \mathrm{~mm}$. The corners of the screw holders are chamfered to increase longevity.

The potential power change depends on the lens surfaces shape. ${ }^{7}$ However, the design can be easily adapted to get the typical power changes needed in eye refraction when the lenses are moved $0.35 \mathrm{~mm}$.

The design enables the lenses to be moved independently upward or downward. Therefore, if the lenses are moved by the same magnitude but in opposite directions, the power change can be achieved without modifying the vertical location of the optical center. The maximum values of vertical movements are $4 \mathrm{~mm}$ downward and $1.5 \mathrm{~mm}$ upward. The upper limit sets the maximum amount of power change that can be obtained without changing the optical center. The inferior limit is larger than the upper in order to achieve the alignment of the optical center relative to the line of sight (pantoscopic angle effect correction).

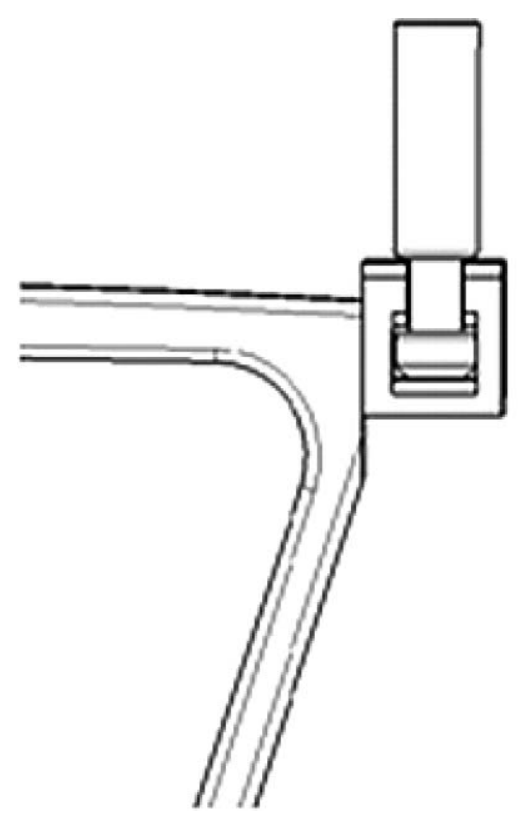

(a)

(b)

Fig. 5 Screws holding the lens holders comprising two parts: (a) threaded and (b) rounded with a circular groove.

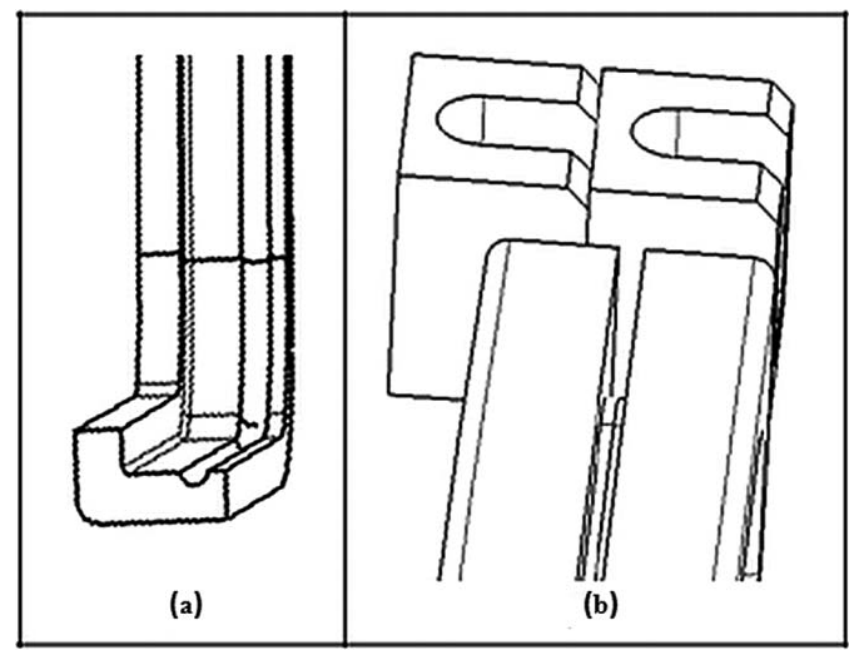

Fig. 6 (a) Inner edge of the lens holder rim. (b) Lens holders of the rear and front lens.

\subsection{Lens Holders and Lens Edges}

The lens holder is a single solid piece comprising two parts: the rims and the screw holders (described previously). Conventionally the lenses are held on the rims by mounting the groove lens edge into the beveled edge of the rim. The beveled edge of the rim is usually created by a mechanization process.

Our design has the advantage of not requiring such mechanization, the spectacles frame can be completely manufactured by injection molding. The lenses were designed with a semicircular lock tab around the edge [Fig. 3(a)] . The rim's inner edge acts as a seat to the lock tab of the lenses [Fig. 6(a)]. The total area of the lens edge is slightly larger $(+0.5 \mathrm{~mm})$ than the area of the rim's seats, so when the lens is mounted into the rim, the difference in area provides the pressure needed to hold the lens in place.

The lateral movement between both lenses is achieved by sliding the two inner planar surfaces of the lenses. There is no gap between the two lenses. The location of the inner surfaces of the rims and the screw holders are not at the same level [Fig. 6(b)], allowing the clamping between the rear and the front lens holders, thus guiding their vertical movement.

\subsection{Spectacle Lens Fixing}

The entire lens frame structure is affixed with the help of several independent pieces specifically designed for each individual part of the frame. The horizontal movement mechanism is affixed under pressure with two upper lids [Fig. 7(a)]. The pressure is achieved by four clips [Fig. 7(b)] located in the lower part of the lids. The clips are set in place following the directions shown in Fig. 7(c). The lids can be dismantled if the replacement of any part of the horizontal movement mechanism is needed.

The lens holders are also affixed under pressure with three front lids [Fig. 8(a)]. These lids have a central shaft to align them with several respective holes located in the fixed structure. They are mounted in the direction shown in Fig. 8(b). Three countersunk screws $(\mathrm{M} 3 \times 13)$ are threaded into these holes [Fig. 9(a)]. 


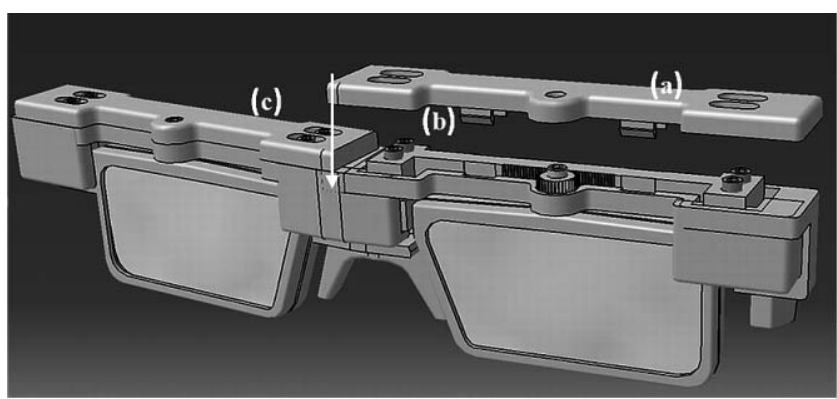

Fig. 7 (a) Upper lids. (b) Clips of upper lids. (c) Mounting direction of upper lids.

\subsection{Frame Sides}

Conventionally, the frame sides are attached to the rims ensuring that they are horizontally aligned to the line joining the pupil centers. However, in our design the lens holders move vertically. Therefore, to accommodate this, the frame sides are attached, not to the lens rims, but to the fixed structure, as shown in Fig. 9. The angle of side and the angle of drop are also considered. ${ }^{14,15}$

Each frame side includes one half of the hinges [Fig. 9(b)]. The other part of the hinges is attached to the fixed part of the frame. Both sides of the hinge are joined with axe screws [Fig. 9(c)].

\section{Spectacle Frame Assembly}

The assembly of all pieces of the spectacle frame has been designed to be performed manually, with the help of just a screwdriver. The assembly is carried out in sequential steps. First, the horizontal movement mechanism: the racks are mounted onto the fixed structure by positioning the guiding mechanized profiles of both structures. The pinions are inserted vertically into two holes located in the fixed structure along their turn shaft [Fig. 1(d)]. Second, the vertical movement mechanism is assembled. The lenses are mounted into the lens holders [following the direction represented in Fig. 3(b)]. Later, four grooved screws, used to fix the rear lens holders, are threaded into the racks [Fig. 2(a)]. The rear lens holders are mounted by matching the screw grooves into the screw holders [Fig. 2(b)]. The same operation is repeated with the front lens holders and screws.

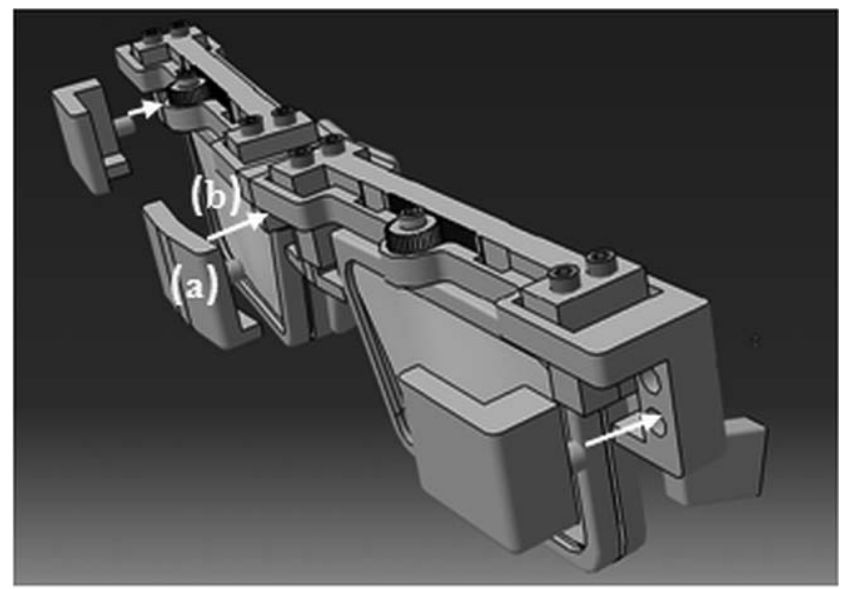

Fig. 8 (a) Front lids. (b) Mounting direction of front lids.

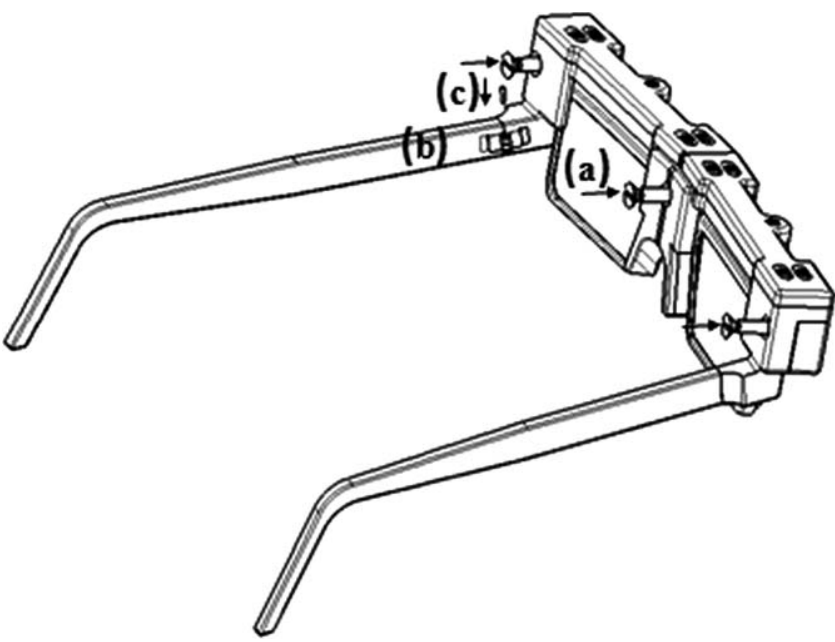

Fig. 9 Rear view of the spectacle frame. (a) Screws fixing the spectacle frame. (b) Frame sides hinges. (c) Axe screws joining the hinges.

The next step is to mount the pieces that fix the frame. The upper lids are mounted in the direction shown in Fig. 7(c), and the front lids align the center shaft with the seat holes on the fixed structure [Fig. 8(b)]. Three screws with countersunk heads are introduced from the back of the fixed structure to the front along the direction indicated in Fig. 9(a). Finally, the frame sides are attached matching the axes of the two halves of the hinges with an axe screw [Fig. 9(c)].

\section{Prototype}

In order to test mechanical functionality, we have built a prototype of the whole spectacle lens frame using fused deposition modeling. This technology constructs three-dimensional objects from CAD generated solid models through a computer numerical controlled machine. Plastic material is laid down in layers with a temperature-controlled head which extrudes the plastic.

The prototype shows empirically that the vertical and horizontal movement mechanism designs work properly. A photograph of the frontal side of the prototype is shown in Fig. 10.

\section{Discussion}

We have presented a new mechanical design of a poweradjustable spectacle lens frame. To our knowledge, this is the

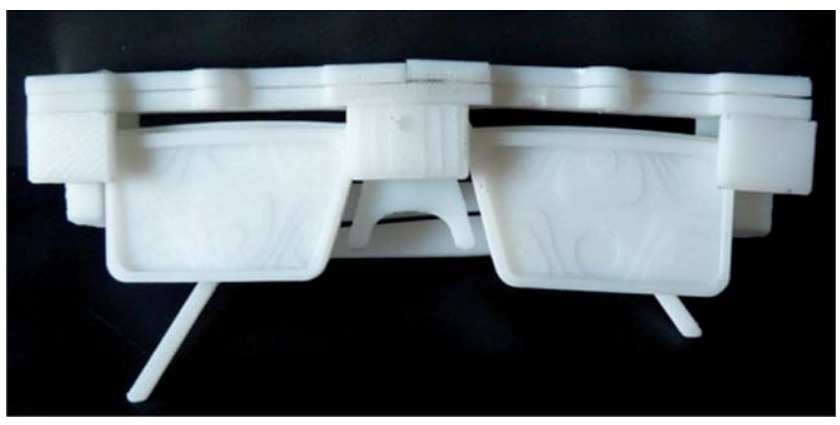

Fig. 10 Front view of the spectacle lens prototype using fused deposition modeling. 
first time that this type of frame has been described in detail in scientific literature; although different design ideas have been published in several patents. Our design was guided by three principles: 1. To provide fine mechanical movements for optical precision. 2. Low cost manufacturing. 3. Easy frame mounting, piece replacement, and cleaning.

The mechanical design provides precise and quantified measures of the lens movement and optical centers distance. Therefore, it is suitable to be used as a device to, not only correct, but also measure refractive errors.

Alvarez-Lohmann lenses have two possible configurations. In the first one, the cubic surfaces are located facing outward, while in the second they are facing inward. ${ }^{7}$ The former implies that a larger overall thickness is needed with respect to the latter in order to avoid the collision between lenses when shifted laterally. In addition, it has been shown ${ }^{7}$ by ray tracing computations that the root mean square error (on-axis) of the spot diagram as a function of power variation is smaller for the inward configuration. In pursuing these benefits we opted for a mechanical design where the plane surfaces are in contact and facing inward.

An innovation of our design, with respect to previous ones, is the addition of an interpupillary matching mechanism. We set a range of maximum horizontal lateral movements of $4 \mathrm{~mm}$. However, this value could be easily increased within the design by reducing the horizontal dimension of the lenses. Obviously, this is a design parameter trade-off. On the one hand, a larger movement range allows matching the interpupillary distance in a larger population, but on the other the horizontal dimension of the lens would be reduced, thus decreasing the horizontal field of view. The final design parameter should depend on the admissible interpupillary distance discrepancy, which in turn depends on the tolerance to horizontal prismatic errors. The prismatic errors generated in Alvarez-Lohmann lenses not only depends on the matching of the interpupillary and the optical center distance, but also on the shape of the surfaces and the lateral shift between lenses. ${ }^{7}$ Future work will be carried out on designing surfaces for Alvarez-Lohmann lenses that minimize prismatic errors.

We note that the interpupillary matching mechanism could also be applied to conventional spectacles. In many cases, the spectacle frames are selected without matching the distance between the lens contours centers and the subject's interpupillary distance. When this occurs, the optical lenses must be cut around a new center, hence setting a nonsymmetric optical surface for the portion of the lens inserted in the lens holder.

The same problem is presented with vertical centering. In conventional spectacle lens dispensing the optical center of lenses are sometimes lowered so that the lens optical axis passes through the center of eye rotation (pantoscopic angle correction). In our design the possibility of moving each pair of lenses vertically (up to $4 \mathrm{~mm}$ downward) allows the control of vertical optical alignment.

Spectacle lens frames are principally manufactured using two different techniques: molding and surfacing. Considering economic cost and the criteria for product quality, it was planned to completely manufacture our design by injection molding. Injection has significant cost benefits, with respect to surfacing, when mass production exceeds a certain amount of units (typically 1000 (Ref. 19) to recover the economical cost of mold manufacturing). A company specialized in plastic injection (MFC Mold
Center S.L.) provided us with the following estimates. The injection molding tools (including the molds) would cost 66,774 euros. The estimated cost of a single unit production is 1.7 euros (with a minimum annual production of 10,000 pieces).

The higher product quality achieved with injection molding, with respect to surfacing, is so because of reduced surface roughness, a higher precision in dimensional tolerances (even with very thin thicknesses, around 0.008 to 0.002 in. ${ }^{19}$ ) and the resultant highly polished and clean surfaces without imperfections that typically appear in surfacing.

For the injection, a thermoplastic or thermosetting material can be employed. However, considering the spectacle lens mount, a thermoplastic material is preferred to a thermosetting one due to it's more stable mechanical properties. We consider acetal resin to be a good choice. In the injection process, this material has high compressive strength, a low coefficient of friction and excellent dimensional stability. ${ }^{20}$ Once the spectacle lens is manufactured, the acetal resin provides high flexural and tensile strength at break, and strength to deformation under load, even at extreme temperatures.

The spectacle lens frame was designed as a modular assembly to facilitate frame mounting, piece replacement (in case of deterioration or breakage), and cleaning of different pieces.

In case lens cleaning is needed, they can be easily removed from the frame by means of dismounting the front lids and holders as shown in Sec. 3. Nevertheless, due to the way the lenses are mounted, without any gap between them, dirt is prevented from being trapped between them. All the pieces are made of plastic, including the screws, hinges, and mobile mechanisms. In the design of all these pieces the surface profiles are angled with respect to the mold cavity surfaces, and the edges of the corners of the pieces are rounded to allow ejection. Finally, an additional advantage of the design is that no subsequent mechanization is needed.

\section{Acknowledgments}

The authors acknowledge funding from CSIC Grant No. N200950I205. The prototype was built at the Instituto de Automatica Industrial and the Instituto de Optica mechanical labs with the help of Javier Sarria, Pedro Peñaranda and Luis A. De Prado. Jorge Fernandez from Mold Center provided estimated costs of manufacturing the spectacle lens frame. We give thanks for the discussions maintained with Antonio Redondo and Jacob Rubinstein, and the overview of the linguistic content to Mandy Darnell and Matthew Glinsman.

\section{References}

1. S. Resnikoff, D. Pascolini, S. P. Mariotti, and G. P. Pokharel, "Global magnitude of visual impairment caused by uncorrected refractive errors in 2004," Bull. World Health Organ. 86, 63-70 (2008).

2. M. G. Douali and J. D. Silver, "Self-optimised vision correction with adaptive spectacle lenses in developing countries," Ophthalmic Physiol. Opt. 24(3), 234-241 (2004).

3. L. Alvarez, "Two-element variable-power spherical lens," US Patent No. 3,305,294 (1967).

4. F. C. v. Asbeck, "Een universele bril (Universal spectacles)," $\mathrm{PhD}$ thesis, Technical University (TU) Delf (2003).

5. G. L. van Der Heijde, "Universal spectacles for children in developing countries," in Mopane: Conference on Visual Optics, Kruger National Park, South Africa (2006). 
6. A. Lohmann, "A new class of varifocal lenses," Appl. Opt. 9(7), 1669-1671 (1970).

7. S. Barbero, "The alvarez and lohmann refractive lenses revisited," $O p t$ Express 17(11), 9376-9390 (2009).

8. W.E. Humphrey, "Remote subjective refractor employing continuously variable sphere-cylinder corrections," Opt. Eng. 15(4), 286-291 (1976).

9. F. C. v. Asbeck, "Combined lens and spectacles provided with at least one such combined lens," International Patent No. WO2006/098618 (2006).

10. B. Spivey, "Adjustable focus eyeglasses," US Patent No. 7,325,922 (2008).

11. G. L. Van Der Heijde and W. P. Louwerse, "Glasses," International Patent No. WO2008/002131 (2008).

12. B. Spivey, "Adjustable focus eyeglasses with pivot adjustments," US Patent No. 2007/030440 (2007)

13. A. W. Dreher and B. Spivey, "Adjustable eyeglasses with a magnetic attachment," International Patent No. WO2009/140255 (2009).
14. C. Y. Tang, N. Tang, and M. C. Stewart, "Facial measurements for frame design," Optom. Vision Sci. 75(4), 288-292 (1998).

15. C. Y. Tang, N. Tang, and M. C. Stewart, "Ophthalmic anthropometry for hong kong chinese adults," Optom. Vision Sci. 75(4), 293-301 (1998).

16. J. Caum Aregay et al., Tecnologa Optica: Lentes Oftalmicas, Diseo y Adaptacion, UPC, Barcelona (1997).

17. G. Walsh, "The weight of spectacle frames and the area of their nose pads," Ophthalmic Physiol. Opt. 30(4), 402-404 (2010).

18. J. S. Pointer, "The far interpupillary distance. a gender-specific variation with advancing age," Ophthalmic Physiol. Opt. 19(4), 317-326 (1999).

19. W. Mink, Practical Injection Moulding of Plastics, Iliffe Books, London (1964).

20. A. Miravete, "Materiales compuestos I," INO Reproducciones S. A., Zaragoza, Spain. 